Article

\title{
A Weighted Estimation Algorithm for Enhancing Pulsed Eddy Current Infrared Image in Ecpt Non-Destructive Testing
}

\author{
Hanchao Li, Yating Yu*D, Linfeng Li and Bowen Liu \\ School of Mechanical and Electrical Engineering, University of Electronic Science and Technology of China, \\ Chengdu 611731, China; lhc@uestc.edu.cn (H.L.); xhullf@163.com (L.L.); lbw9449@163.com (B.L.) \\ * Correspondence: wzwyyt@uestc.edu.cn; Tel.: +86-136-7813-9939
}

Received: 31 July 2019; Accepted: 13 September 2019; Published: 9 October 2019

check for updates

\begin{abstract}
Non-destructive testing (NDT) plays a crucial role in large scale industrial production such as in the nuclear industry and bridge structures where even a small crack can lead to severe accidents. The pulsed eddy current infrared thermography testing method, as a classic non-destructive testing technology, is proposed to detect cracks in the presence of excitation sources that cause temperature changes in the vicinity of defects, which is higher than normal area. However, in the vicinity of the excitation sources, the temperature is higher than normal even if there is no defect. Traditional infrared image enhancing algorithms do not work efficiently when processing infrared images because the colors in the images represent the temperature. To address this, a novel algorithm is proposed in this paper. A weighted estimation algorithm is proposed because each pixel value has a strong relationship with its neighboring pixels. The value of each pixel is determined by calculating the values of its neighboring pixels with a specific step-size and the correlation coefficients between them. These coefficients are obtained by calculating the differences between the pixels. The experimental results indicated that the outline of the welding defect became significantly clearer after being processed using the proposed algorithm, which can eliminate the errors caused by the excitation source.
\end{abstract}

Keywords: non-destructive testing evaluation; infrared thermography testing; defect detection; image enhancement

\section{Introduction}

Defects detection such as in bridges and nuclear structures testing, especially the small and surface/subsurface cracks detection, is the main target of non-destructive testing (NDT) [1-3]. These kinds of defects such as welding defect, which can cause severe accidents in large scale industrial production and transportation, have been studied for many years [4]. The application of new materials for industrial processes make it difficult to detect defects using traditional NDT methods such as eddy current testing (ECT) technology. For instance, with the application of new materials in modern aircraft manufacturing, composites are widely used to manufacture the key components of the aircraft. As a consequence, infrared thermography testing which is an important branch of NDT [5-7], is gaining attention as an efficient method for defect detection in new materials [8-10]. Compared to other NDT technologies, infrared thermography testing has significant advantages. It can be used to detect defects for various materials and geometries [11]. It can also be used for the detection and location of wielding defects by collecting and processing infrared images [12-15].

Infrared thermography testing technology produces images that show the temperature distribution of the defects and the background [16].

The images are processed to collect as much information as possible to reconstruct the defects. Many researchers have done considerable amounts of work on the processing of infrared images [17,18]. 
The image processing approach can be classified into two categories namely spatial and frequency domain methods.

In spatial domain infrared image processing methods, the pixels of the image are manipulated directly. This is done by performing mathematical operations on each pixel and the surrounding pixels to obtain the gray value of the pixel. Common spatial domain image processing methods utilize sharp and smooth filters [19-21]. The fundamental purpose of the spatial domain processing method is to distinguish the defects from the background.

The frequency domain method transforms the image into frequency domain using a frequency transform methods such as the Fourier transform (FT) or wavelet transform (WT) [22]. The first step of the frequency domain image processing method is to convert the image into the frequency domain, after which the signal in the frequency domain is filtered. The frequency domain method yields low and high frequencies, which can be distinguished by a frequency transform of the infrared image. The low and the high frequency components are enhanced and suppressed respectively, such that the information of the defects in the image is made clearer.

However, most of the image processing methods only focus on the image itself, ignoring the significant differences between infrared and common images. In particular, infrared images do not show the actual scene being imaged. Instead, they show the distribution of temperature, which is influenced by excitation sources. In this paper, a method for enhancing infrared images is proposed. This method can eliminate the influence of the excitation source, and yield an infrared image with a clearer defect profile.

The essence of infrared thermography non-destructive testing technology is to obtain the temperature by measuring the amount of infrared radiation from the surface of an object. The common excitation sources of infrared thermography testing can be classified into three types: optical, electromagnetic, and mechanical excitation sources. In electromagnetic excitation sources, eddy current pulsed thermography (ECPT) is widely used as it can uniformly generate sufficient heat in a short time, and the heat it provides is generated from the eddy current induced from the excitation source. This allows the object to be heated evenly and makes the outline of the defect in the infrared image clearer [23].

The operating principle of eddy current pulsed thermography can be explained as follows. A high-frequency alternating current is applied to the excitation coil, which induces an eddy current on the surface and inside the conductive object. The inducted eddy current flows in the conductive object. The conduction of the eddy current is influenced by the size, shape, and position of the defect, as the eddy current is forced to flow around the defect [24].

Figure 1 shows the spreading process of the eddy current in an object containing a defect. The distribution of the eddy current is influenced by the defect. The eddy current is much denser the edge of the defect than it is in other regions without defects.

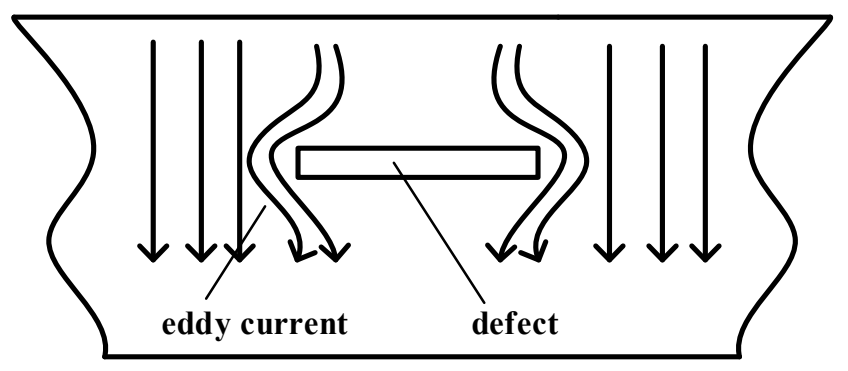

Figure 1. Eddy current at the edge of the defect.

The distribution of heat generated according to Joule's Law is uniform, because the distribution of eddy current is uniform. Joule's law is as follows:

$$
Q=\frac{1}{\sigma}|J|^{2} t
$$


where $\sigma$ is the electrical conductivity of the object and $J$ is the eddy current density, $t$. It is known from Joule's Law that the heat generated increases as the eddy current density increases.

An infrared thermal imager can detect and record thermal radiation on the surface and display it in the form of a temperature value in the infrared image. Figure 2 shows a schematic of the operating principle of the infrared thermal imager.

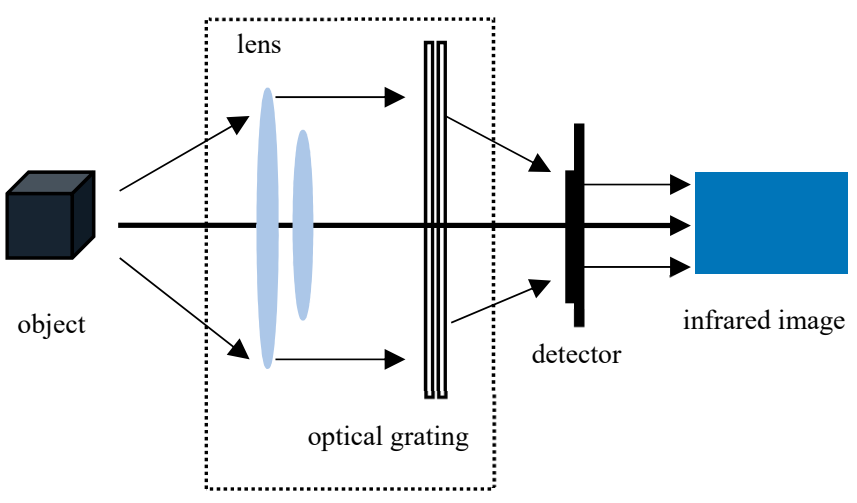

Figure 2. Operating principle of infrared thermal imager.

The different colors in the infrared image show the different temperatures on the surface of the object. A brighter region in the infrared image corresponds to a higher temperature on the surface of the detected object. The presence of an excitation source causes the infrared image collected in the experiment to differ from an image obtained under ideal conditions. However, most researchers have ignored the influence of the excitation source. An excitation source has a significant influence on an infrared image. The temperature of the surface in the presence of an excitation source is significantly higher than that of the surface far from the excitation source. We can find that there is a huge part whose size and shape is similar to the excitation source different from the background in the infrared image. Typically, the region influenced by the excitation source appears brighter in the infrared image, which means that the temperature is higher than the background without a defect underneath. If the defect is directly under the location of the excitation source on the surface, it is difficult to distinguish the defect. The area in the white box in Figure 3 is directly under the excitation source coil. There is a defect under the surface in the left box containing brighter colors. However, it is difficult to determine whether there is a defect inside the area in the right box, because its color is too similar to the background.

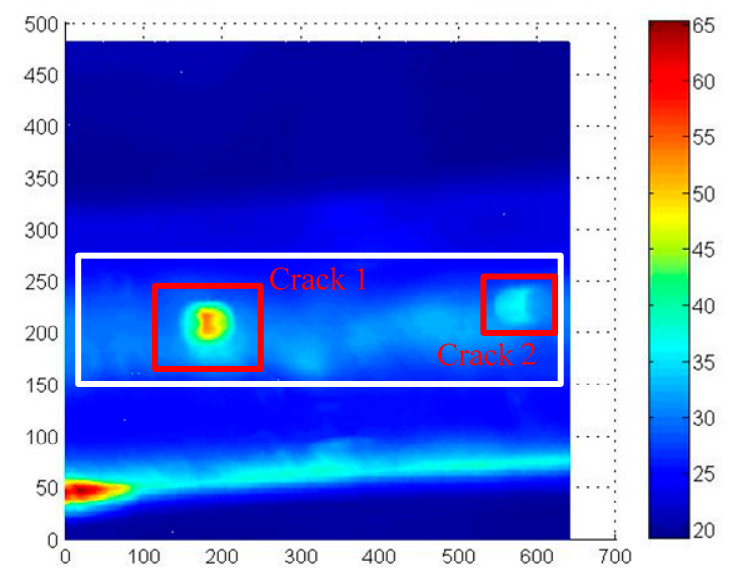

Figure 3. Infrared image.

The infrared image was meshed to analyze the temperature in the image. Figure 4 shows the temperature distribution of the image shown in Figure 3. 


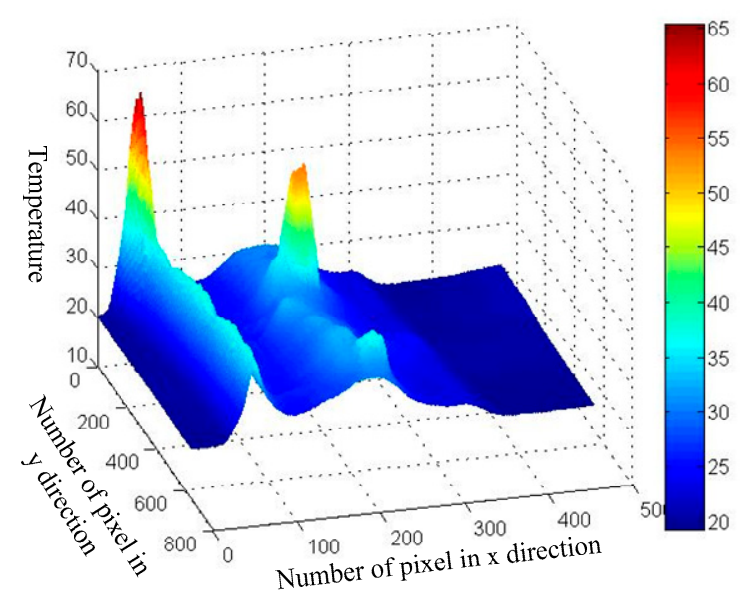

Figure 4. Meshed infrared image.

The temperature in the area directly beneath the excitation source was higher than that in other areas, causing a temperature swell to appear. The highest swell was caused by the defect in the left box in Figure 3. However, the swell caused by the defect in the right box in Figure 3 is not as clear because the excitation source is too large.

To eliminate the influence of the excitation source and make the defect profile clearer in the infrared image, several processing operations are proposed in this paper.

Several operations were applied to the pixels in the infrared image. We considered the value of a target pixel in the infrared image to be influenced by the neighboring pixels. The neighboring eight pixels were chosen to obtain the value of the target pixel.

\section{Experiment Setup}

In the infrared thermal imaging experiment, the specimen was heated to create different thermal distributions in the cracked and undamaged areas. A pulsed eddy current was applied to heat the material from the inside. In this way, the cracked area in the specimen produced considerably more heat than other areas. Generally, cracks distributed at different depths can be detected by NDT methods. The heat information at different depths depends on the rich frequency components during the pulsed eddy current excitation. Deep cracks could not be detected using infrared thermal imaging testing, because the heat transfer distribution became uniform over time as the heat was transferred from deeper regions to the surface. The experimental setup is shown in Figure 5.

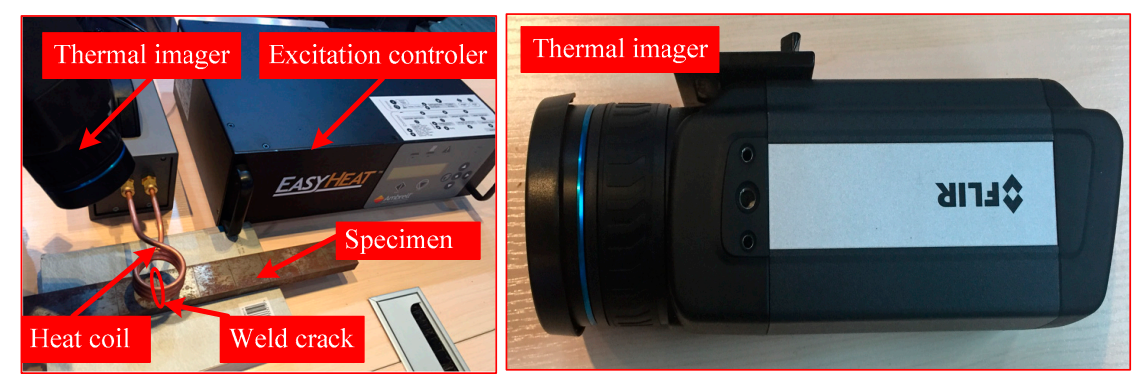

Figure 5. Experimental setup.

Figure 5 shows that the excitation coil was restricted by the excitation controller. To detect deep cracks, the period of the square wave signal could be varied by the excitation controller. The specimen used in the experiment was a ferromagnetic material, and the cracks in it were artificial defects with depths of $0.1,0.8$, and $3 \mathrm{~mm}$ and width of $0.5 \mathrm{~mm}$. The thermal imager and excitation controller could be simultaneously controlled by the computer (PC), where a $100 \mathrm{~ms}$ heating duration is selected for 
inspection. This heating time is long enough to elicit an observable heat pattern. The excitation coil is $60 \mathrm{~mm}$ width placed above the specimen, and the distance between the thermal imager and specimen is about $600 \mathrm{~mm}$. The signal flow chart in the experiment is shown in Figure 6.

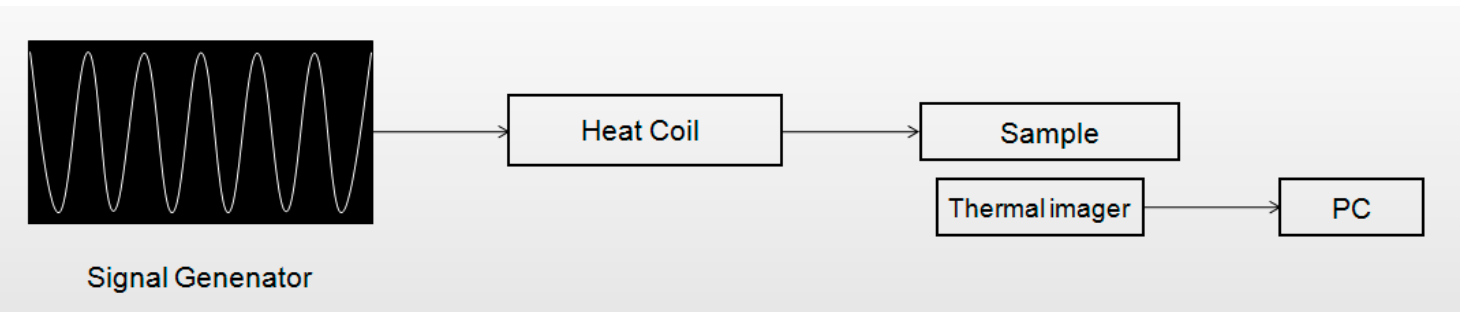

Figure 6. Signal flow chart.

\section{Proposed Algorithm}

The coordinate of the selected pixel is $(i, j)$. The pixel value is denoted as $F(i, j)$. The values of the neighboring eight pixels with the specified step-sizes are as follows: $F(i-s, j-s), F(i-s, j)$, $F(i-s, j+s), F(i, j-s), F(i, j+s), F(i+s, j-s), F(i+s, j)$, and $F(i+s, j+s)$, which can be expressed in matrix form as follows:

$$
P=\left\{\begin{array}{l}
F(i-s, j-s) ; \\
F(i-s, j) ; \\
F(i-s, j+s) ; \\
F(i, j-s) ; \\
F(i, j+s) ; \\
F(i+s, j-s) ; \\
F(i+s, j) ; \\
F(i+s, j+s) ;
\end{array}\right\},
$$

The model of the pixels in the infrared image is shown in Figure 7. The pixel value of a selected pixel, $F(i, j)$ (yellow box in Figure 7), is considered to be related to the neighboring eight pixels, whose step-sizes are 2 (green boxes in Figure 7). For convenience, the neighboring pixels are expressed by $a_{i}$, arranged in clockwise order from the top left corner.

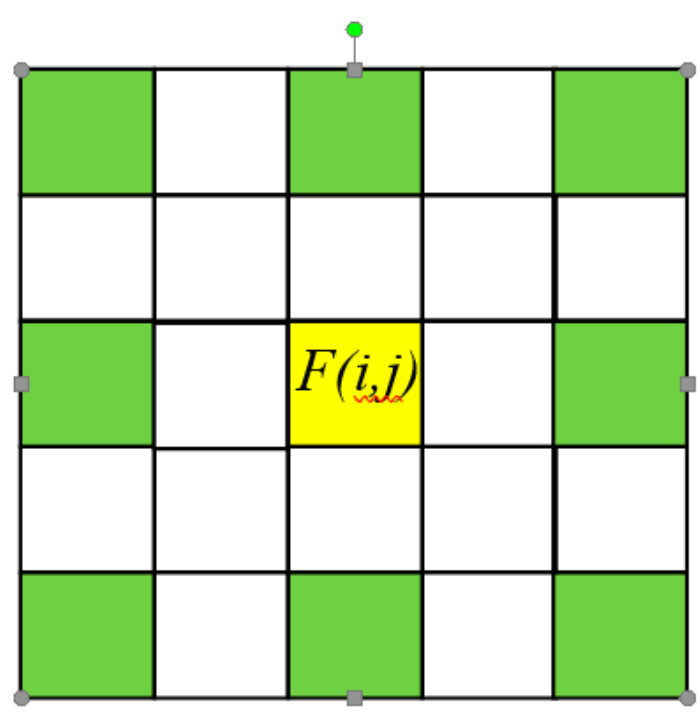

Figure 7. Model of pixels in infrared image. 
To find the relationships between $F(i, j)$ and the neighboring pixels, we subtract the values of neighboring pixels from the value of $F(i, j)$ and arrange them in a column matrix, $L$, as follows:

$$
L=\left\{\begin{array}{l}
X(i, j)-a_{1} \\
X(i, j)-a_{2} \\
X(i, j)-a_{3} \\
X(i, j)-a_{4} \\
X(i, j)-a_{5} \\
X(i, j)-a_{6} \\
X(i, j)-a_{7} \\
X(i, j)-a_{8}
\end{array}\right\},
$$

The differences between $F(i, j)$ and the neighboring pixels shows the correlations between them. The correlation matrix, $R$, representing the correlation between the neighboring pixels is obtained from the difference matrix, $L$, as follows:

$$
R=L \times L^{T} ，
$$

The differences between $F(i, j)$ and the neighboring pixels are denoted as $\Delta_{1}-\Delta_{8}$. After calculating the correlation between $F(i, j)$ and the neighboring pixels, the value of $F(i, j)$ is calculated as follows:

$$
F(i, j)=\sqrt{\sum_{i=1}^{8} \sum_{j=1}^{8} \Delta_{i} \Delta_{j} a_{i} a_{j}},
$$

Equation (5) can be written as follows:

$$
F(i, j)=\sqrt{P^{T} \times R \times P},
$$

The value of $F(i, j)$ obtained from the process above is not the exact value, as the sum of the correlation coefficients is not equal to 1 . Thus, to obtain the real value of $F(i, j)$, the value above must be normalized as follows:

$$
F(i, j)=\frac{F(i, j)}{\left(\sum_{i=1}^{n} \Delta_{i}\right)^{2}}
$$

The infrared image can finally be obtained by calculating the value of every pixel using the method presented above.

\section{Results and Discussion}

The infrared image analyzed is shown in Figure 3. The image was collected with the excitation source directly above the surface and two defects under it. The defect on the right side of the image is not clear.

By verifying the value of the step-size, we determined that the method was most efficient when the step-size was 8 . Figure 8 shows the result when the step-size was 8 . Compared with the initial infrared image, it is evident that the swell caused by the excitation source was eliminated.

Figure 9 shows the contrast between the processed infrared image and the initial image. The infrared image after processing was significantly clearer than the initial image. The bright area influenced by the excitation source was eliminated in the processed infrared image. Furthermore, the bright area in the right box was caused by the defect inside the object became clearer. 


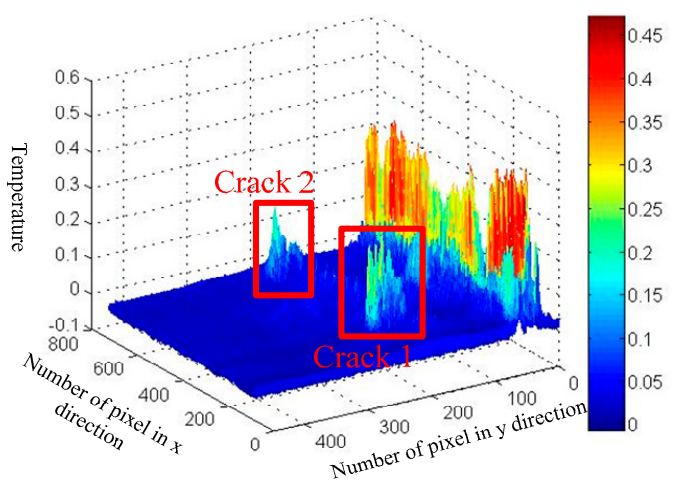

Figure 8. Meshed infrared image after processing.

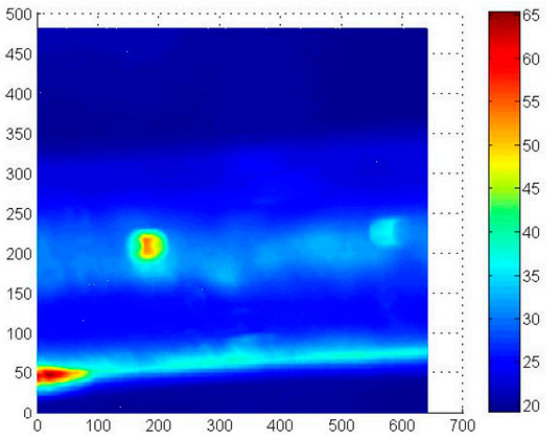

(a)

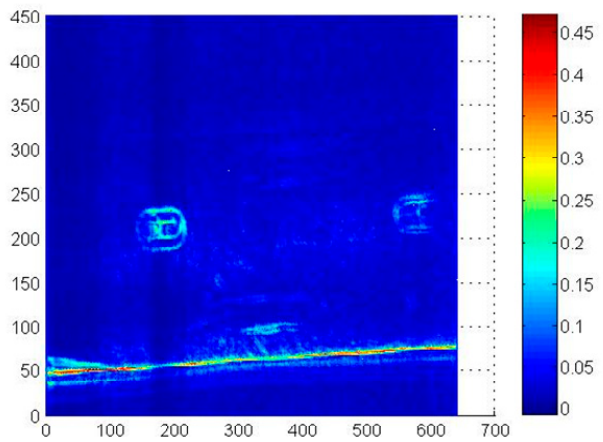

(b)

Figure 9. Comparison of initial and processed images: (a) Initial infrared image; (b) Infrared image after processing.

The swell in the infrared image caused by the excitation source was eliminated by using the algorithm provided in this paper. Furthermore, the outlines of the defects were made more distinct. The method presented in this paper improved the defect detection accuracy by enhancing the two crack images, and most importantly, it reduced the background noise which could increase the credibility of crack estimation compared with the original detected infrared image.

It should be noted that this algorithm is designed to reduce the background noise imported through the temperature of the environment and the excitation. Furthermore, in the pulsed eddy current infrared imaging test, multiple cracks will influence the distribution of the temperature so that the low temperature area of a crack will be covered in the background. This is because the thermal imager should adapt to the temperature in the whole vision area to make sure the image is clear. Thus, the proposed method is motivated by this problem. From the results, it can be inferred that when multiple cracks exist in the visible area, this method could reduce the influences produced by the thermal transforming. At stable temperatures, because the temperature between every two crack is similar and smooth but the temperature in the crack area is higher than that of the other areas, this method could be used.

\section{Conclusions}

A novel algorithm was proposed to enhance the infrared images by performing mathematical operations using pixel values with a specific step-size for the detection of welding defects. The pixel value was calculated using the neighboring pixels' values and the correlation between them. The relationship between the neighboring pixels was inferred from the differences between the target and neighboring pixel values. The correlation coefficients between the neighboring pixels were arranged in a matrix. The elements were multiplied by the corresponding pixel values and were subsequently summed and normalized, yielding a final pixel value. The method proposed in this paper 
represents each pixel value using the neighboring pixels, eliminating swells in an infrared image due to an excitation source, which make the detection of defects significantly more difficult. The outline of the defect was found to be clearer when the processing was performed using the above-mentioned method.

Author Contributions: H.L. contributes to most of the results including modeling construction and analysis of the results, and he also completes the English writing of this paper. Y.Y. proposed the main idea of this article. L.L. and B.L. offer some help in processing data and draw diagrams.

Funding: This work is financial supported by the Fundamental Research Funds for the Central Universities under Grant ZYGX2018J067 and the Nature Science Foundation of Guangdong Province under Grant 2018A030313893.

Conflicts of Interest: The authors declare no conflicts of interest.

\section{References}

1. Ebrahimkhanlou, A.; Athanasiou, A.; Hrynyk, T.D.; Bayrak, O.; Salamone, S. Fractal and Multifractal Analysis of Crack Patterns in Prestressed Concrete Girders. J. Bridge Eng. 2019, 24, 04019059. [CrossRef]

2. Ebrahimkhanlou, A.; Salamone, S.; Ebrahimkhanlou, A.; Azad, A.R.G.; Kreitman, K.; Helwig, T.; Williamson, E.; Engelhardt, M. Acoustic emission monitoring of strengthened steel bridges: Inferring the mechanical behavior of post-Installed shear connectors. In Proceedings of the Nondestructive Characterization and Monitoring of Advanced Materials, Aerospace, Civil Infrastructure, and Transportation XIII, Denver, CO, USA, 4-7 March 2019.

3. Ebrahimkhanlou, A.; Choi, J.; Hrynyk, T.D.; Salamone, S.; Bayrak, O. Detection of the onset of delamination in a post-Tensioned curved concrete structure using hidden Markov modeling of acoustic emissions. In Proceedings of the Sensors and Smart Structures Technologies for Civil, Mechanical, and Aerospace Systems 2018, Anaheim, CA, USA, 26-30 April 2018.

4. Tian, L.; Cheng, Y.; Yin, C.; Ding, D.; Song, Y.; Bai, L. Design of the MOI method based on the artificial neural network for crack detection. Neurocomputing 2017, 226, 80-89. [CrossRef]

5. Park, H.; Choi, M.; Park, J.; Kim, W. A study on detection of micro-Cracks in the dissimilar metal weld through ultrasound infrared thermography. Infrared Phys. Technol. 2014, 62, 124-131. [CrossRef]

6. Rodríguez-Martin, M.; Lagüela, S.; González-Aguilera, D.; Arias, P. Cooling analysis of welded materials for crack detection using infrared thermography. Infrared Phys. Technol. 2014, 67, 547-554. [CrossRef]

7. Dapeng, C.; Hongxia, M.; Zhihe, X. Infrared Thermography NDT and Its Development. Comput. Meas. Control 2016, 4, 1-6.

8. Plotnikov, Y.A.; Winfree, W.P. Advanced Image Processing for Defect Visualization in Infrared Thermography. Proc. SPIE 1998, 3361, 331-338.

9. Liu, Z.P.; Hu, L.H.; Zhou, J.M.; Cai, L. Evaluation of Surface Defect Area in Metal Based on Infrared Thermal Image. Appl. Mech. Mater. 2014, 530, 171-174. [CrossRef]

10. Min, Q.X.; Feng, F.Z.; Wang, P.F.; Zhang, C.S.; Zhu, J.Z. Recognition of contact interface defect in metal plate based on pulsed phase thermography. In Proceedings of the Prognostics \& System Health Management Conference, Beijing, China, 21-23 October 2015.

11. Fan, C.F.C.; Sun, F.S.F.; Yang, L.Y.L. A general quantitative identification algorithm of subsurface defect for infrared thermography. In Proceedings of the 2005 Joint 30th International Conference on Infrared and Millimeter Waves and 13th International Conference on Terahertz Electronics, Williamsburg, VA, USA, 19-23 September 2005.

12. Meola, C.; Carlomagno, G.M. Recent advances in the use of infrared thermography. Meas. Sci. Technol. 2004, 15, 27. [CrossRef]

13. Maldague, X. Applications of infrared thermography in nondestructive evaluation. In Trends in Optical Nondestructive Testing; Elsevier Science: Amsterdam, NL, USA, 2000; pp. 591-609.

14. Avdelidis, N.P.; Hawtin, B.C.; Almond, D.P. Transient thermography in the assessment of defects of aircraft composites. Ndt E Int. 2003, 36, 433-439. [CrossRef]

15. Wang, X. Transient Thermography for Detection of Micro-Defects in Multilayer Thin Films. Ph.D. Thesis, Loughborough University, Loughborough, UK, 2017. 
16. Chen, D.; Zhang, X.; Zhang, G.; Zhang, Y.; Li, X. Infrared Thermography and Its Applications in Aircraft Non-destructive Testing. In Proceedings of the 2016 International Conference on Identification, Information and Knowledge in the Internet of Things (IIKI), Beijing, China, 20-21 October 2016.

17. Li, H.J.; Lin, J.G.; Mei, X.; Zhao, Z. Infrared Image Denoising Algorithm Based on Adaptive Threshold NSCT. In Proceedings of the 2008 Congress on Image and Signal Processing, Sanya, China, 27-30 May 2008.

18. Ibarra-Castanedo, C.; Gonzalez, D.; Klein, M.; Pilla, M.; Vallerand, S.; Maldague, X. Infrared image processing and data analysis. Infrared Phys. Technol. 2004, 46, 75-83. [CrossRef]

19. Wang, J.; Hong, J. A new self-Adaptive weighted filter for removing noise in infrared images. In Proceedings of the 2009 International Conference on Information Engineering and Computer Science, Wuhan, China, 9-20 December 2009.

20. Voronin, V.; Tokareva, S.; Semenishchev, E.; Agaian, S. Thermal Image Enhancement Algorithm Using Local and Global Logarithmic Transform Histogram Matching with Spatial Equalization. In Proceedings of the 2018 IEEE Southwest Symposium on Image Analysis and Interpretation (SSIAI), Las Vegas, NV, USA, 4-10 April 2018.

21. Bai, J.; Chen, Q.; Wang, X.; Qian, W. Contrast enhancement algorithm of infrared image based on noise filtering model. Infrared Laser Eng. 2010, 39, 777-780.

22. Liu, T.; Zhang, W.; Yan, S. A novel image enhancement algorithm based on stationary wavelet transform for infrared thermography to the de-bonding defect in solid rocket motors. Mech. Syst. Signal Process. 2015, 62, 366-380. [CrossRef]

23. Yin, A.; Gao, B.; Yun Tian, G.; Woo, W.L.; Li, K. Physical interpretation and separation of eddy current pulsed thermography. J. Appl. Phys. 2013, 113, 064101. [CrossRef]

24. He, Y.; Pan, M.; Luo, F. Defect characterisation based on heat diffusion using induction thermography testing. Rev. Sci. Instrum. 2012, 83, 104702. [CrossRef]

(C) 2019 by the authors. Licensee MDPI, Basel, Switzerland. This article is an open access article distributed under the terms and conditions of the Creative Commons Attribution (CC BY) license (http://creativecommons.org/licenses/by/4.0/). 